\title{
STANLEY-REISNER RINGS, SHEAVES, AND POINCARÉ-VERDIER DUALITY
}

\author{
KOHJI YANAGAWA
}

\begin{abstract}
A few years ago, I defined a squarefree module over a polynomial ring $S=k\left[x_{1}, \ldots, x_{n}\right]$ generalizing the Stanley-Reisner ring $k[\Delta]=S / I_{\Delta}$ of a simplicial complex $\Delta \subset 2^{\{1, \ldots, n\}}$. This notion is very useful in the StanleyReisner ring theory. In this paper, from a squarefree $S$-module $M$, we construct the $k$-sheaf $M^{+}$on an $(n-1)$ simplex $B$ which is the geometric realization of $2^{\{1, \ldots, n\}}$. For example, $k[\Delta]^{+}$is (the direct image to $B$ of) the constant sheaf on the geometric realization $|\Delta| \subset B$. We have $H^{i}\left(B, M^{+}\right) \cong\left[H_{\mathrm{m}}^{i+1}(M)\right]_{0}$ for all $i \geq 1$. The Poincaré-Verdier duality for sheaves $M^{+}$on $B$ corresponds to the local duality for squarefree modules over $S$. For example, if $|\Delta|$ is a manifold, then $k[\Delta]$ is a Buchsbaum ring and its canonical module $K_{k[\Delta]}$ is a squarefree module which gives the orientation sheaf of $|\Delta|$ with the coefficients in $k$.
\end{abstract}

\section{Introduction}

This paper presents a new geometric aspect of combinatorial commutative algebra on normal semigroup rings. But, in this introduction, we restrict ourselves to the polynomial ring case for simplicity. In this paper, we use the theory of sheaves on a locally compact topological space. For this theory, consult [I]. Basically, we use the same notation as [I] here.

Let $S=k\left[x_{1}, \ldots, x_{n}\right]$ be a polynomial ring over a field $k$, and $\Delta$ a simplicial complex whose vertex set is a subset of $[n]:=\{1, \ldots, n\}$. Then the StanleyReisner ring $k[\Delta]:=S /\left(\prod_{i \in F} x_{i} \mid F \subset[n]\right.$ with $\left.F \notin \Delta\right)$ of $\Delta$ reflects topological properties of the geometric realization $|\Delta|$, and has been studied since 1970's (see [BH, Sta]). In [Y1], the author introduced the notion of a squarefree module which generalizes Stanley-Reisner rings. This notion allows us to apply homological methods (e.g., derived categories) to the theory of Stanley-Reisner rings more systematically. The purpose of this paper is to give a geometric meaning of squarefree modules.

Let $B$ be an $(n-1)$-simplex which is the geometric realization of $2^{[n]}$. We construct the $k$-sheaf $M^{+}$on $B$ from a squarefree module $M$. For example, $k[\Delta]^{+} \cong j_{*} \underline{k}_{|\Delta|}$, where $\underline{k}_{|\Delta|}$ is the constant sheaf on $|\Delta|$ and $j:|\Delta| \rightarrow B$ is the embedding map. Let Sq be the category of squarefree modules, and $\operatorname{Sh}(B)$ the category of $k$-sheaves on $B$. Then the functor $(-)^{+}: \mathrm{Sq} \rightarrow \mathrm{Sh}(B)$ is exact.

Received February 14, 2003. 
If $M$ is a squarefree $S$-module, Theorem 3.3 gives an isomorphism

$$
H^{i}\left(B, M^{+}\right) \cong\left[H_{\mathfrak{m}}^{i+1}(M)\right]_{0} \text { for all } i \geq 1,
$$

and an exact sequence

$$
0 \rightarrow\left[H_{\mathfrak{m}}^{0}(M)\right]_{0} \rightarrow M_{0} \rightarrow H^{0}\left(B, M^{+}\right) \rightarrow\left[H_{\mathfrak{m}}^{1}(M)\right]_{0} \rightarrow 0
$$

where $H_{\mathfrak{m}}^{i}(-)$ stands for the local cohomology module with support in the maximal ideal $\mathfrak{m}:=\left(x_{1}, \ldots, x_{n}\right)$. So our functor $(-)^{+}$is somewhat analogous to "Proj" of the scheme theory.

If $\mathbf{a}=\left(a_{1}, \ldots, a_{n}\right) \notin-\mathbb{N}^{n}$, it is well-known that $\left[H_{\mathfrak{m}}^{i}(M)\right]_{\mathbf{a}}=0$. If $0 \neq$ $\mathbf{a} \in-\mathbb{N}^{n},\left[H_{\mathfrak{m}}^{i}(M)\right]_{\mathbf{a}}$ is isomorphic to the cohomology with compact support $H_{c}^{i-1}\left(U_{F}, j^{*} M^{+}\right)$, where $U_{F}$ is the open subset determined by $F:=\{i \in[n] \mid$ $\left.a_{i}<0\right\}$ (see Theorem 3.5 for detail) and $j: U_{F} \rightarrow B$ is the embedding map. These results generalize a well-known formula of Hochster on $H_{\mathfrak{m}}^{i}(k[\Delta])$.

Let $D^{b}(\mathrm{Sq})$ be the bounded derived category of Sq and $\omega_{S}^{\bullet} \in D^{b}(\mathrm{Sq})$ an injective resolution of $K_{S}[n-1]$. Set $\omega_{k[\Delta]}^{\bullet}:=\operatorname{Hom}_{k[\Delta]}^{\bullet}\left(k[\Delta], \omega_{S}^{\bullet}\right)$. Then $\omega_{k[\Delta]}^{\bullet}$ is a complex of squarefree $k[\Delta]$-modules, and isomorphic to a (non-normalized) $\mathbb{Z}^{n}$-graded dualizing complex of $k[\Delta]$ in the derived category. Let $\mathcal{D}_{|\Delta|}^{\bullet}$ be a dualizing complex of the topological space $|\Delta|$ with the coefficients in $k$. Corollary 4.3 states that $\mathcal{D}_{|\Delta|}^{\bullet} \cong j^{*}\left(\omega_{k[\Delta]}^{\bullet}\right)^{+}$in $D^{b}(\operatorname{Sh}(|\Delta|))$, where $j:|\Delta| \rightarrow B$ is the embedding map. (Since the functors $(-)^{+}: \operatorname{Sq} \rightarrow \operatorname{Sh}(B)$ and $j^{*}: \operatorname{Sh}(B) \rightarrow \operatorname{Sh}(|\Delta|)$ are exact, we have the functor $j^{*}(-)^{+}: D^{b}(\mathrm{Sq}) \rightarrow D^{b}(\operatorname{Sh}(|\Delta|))$.) Moreover, if each component $M^{i}$ of $M^{\bullet} \in D^{b}(\mathrm{Sq})$ is a $k[\Delta]$-module, we have

$$
R \mathcal{H o m}_{\mathrm{Sh}(|\Delta|)}\left(j^{*}\left(M^{\bullet}\right)^{+}, \mathcal{D}_{|\Delta|}^{\bullet}\right) \cong j^{*}\left(R \operatorname{Hom}_{k[\Delta]}\left(M^{\bullet}, \omega_{k[\Delta]}^{\bullet}\right)^{+}\right)
$$

in $D^{b}(\operatorname{Sh}(|\Delta|))$. See Theorem 4.2. If further $\left[H^{i}\left(M^{\bullet}\right)\right]_{0}=0$ for all $i$ (note that the sheaf $M^{+}$does not reflect the degree 0 component $M_{0}$ ), we have

$$
\operatorname{Ext}_{\operatorname{Sh}(|\Delta|)}^{i}\left(j^{*}\left(M^{\bullet}\right)^{+}, \mathcal{D}_{|\Delta|}^{\bullet}\right) \cong\left[\operatorname{Ext}_{k[\Delta]}^{i}\left(M^{\bullet}, \omega_{k[\Delta]}^{\bullet}\right)\right]_{0}
$$

So the Poincaré-Verdier duality for $|\Delta|$ corresponds to the local duality for $k[\Delta]$ in our context. For example, if $|\Delta|$ is a manifold, then $k[\Delta]$ is a Buchsbaum ring and the canonical module $K_{k[\Delta]}$ of $k[\Delta]$ is a squarefree module which gives the orientation sheaf of $|\Delta|$ with the coefficients in $k$. So, the well-known duality between $H_{\mathfrak{m}}^{i}(k[\Delta])$ and $H_{\mathfrak{m}}^{j}\left(K_{k[\Delta]}\right)$ ([SV, II. Theorem 4.9]) corresponds to the Poincaré duality for $|\Delta|$.

\section{Preliminaries}

Let $Q \subset \mathbb{N}^{n}$ be an affine semigroup (i.e., a finitely generated sub-semigroup containing 0 ), and $k[Q]=k\left[x^{\mathbf{a}} \mid \mathbf{a} \in Q\right] \subset S:=k\left[x_{1}, \ldots, x_{n}\right]$ the semigroup ring of $Q$ over a field $k$. Here $x^{\mathbf{a}}$ for $\mathbf{a}=\left(a_{1}, \ldots, a_{n}\right) \in \mathbb{N}^{n}$ means the monomial $\prod x_{i}^{a_{i}} \in S$. We always assume that $Q$ is saturated (i.e., if $\mathbf{a} \in \mathbb{N}^{n}$ satisfies $m \mathbf{a} \in Q$ for a positive integer $m$, then $\mathbf{a} \in Q)$ and $\mathbb{Z} Q=\mathbb{Z}^{n}$. Thus $k[Q]$ is a normal Cohen-Macaulay $\mathbb{Z}^{n}$-graded ring of dimension $n$ with the graded maximal 
ideal $\mathfrak{m}=\left(x^{\mathbf{a}} \mid 0 \neq \mathbf{a} \in Q\right)$. For basic properties of ( $\mathbb{Z}^{n}$-graded modules over) $k[Q]$ and the related notions from convex geometry, see [BH, GW].

Consider the polyhedral cone $\mathbb{R}_{\geq 0} Q \subset \mathbb{R}^{n}$ spanned by $Q\left(\subset \mathbb{N}^{n} \subset \mathbb{R}^{n}\right)$. Let $L$ be the set of non-empty faces of $\mathbb{R}_{\geq 0} Q$. The order by inclusion makes $L$ a finite poset. If $p \in \mathbb{R}_{>0} Q$, there is a unique face $F \in L$ such that the relative interior rel-int $(F)$ of $F$ contains $p$. We call this $F$ the support of $p$, and denote it by $\operatorname{supp}(p)$.

If $\mathbb{R}_{\geq 0} Q$ is spanned by $n$ vectors as a polyhedral cone, we say $k[Q]$ is simplicial. In this case, $L$ is isomorphic to the boolean lattice $2^{[n]}$ as a poset. For example, the polynomial ring $k\left[\mathbb{N}^{n}\right]=k\left[x_{1}, \ldots, x_{n}\right]$ is a simplicial semigroup ring.

Let $H$ be a hyperplane of $\mathbb{R}^{n}$ which intersects the cone $\mathbb{R}_{\geq 0} Q$ transversally. Consider the $(n-1)$-dimensional polytope $B:=H \cap \mathbb{R}_{\geq 0} Q$. Of course, $B$ is homeomorphic to a closed ball of dimension $n-1$. If $k[Q]$ is simplicial, then $B$ is a simplex. For a face $F \in L$, set $|F|:=F \cap H$ to be a face of $B$, and $|F|^{\circ}:=\operatorname{rel}-i n t(|F|)$ its relative interior. If $\Delta \subset L$ is an order ideal (i.e., $F \in \Delta$, $G \in L$ and $F \supset G \Rightarrow G \in \Delta)$, then $|\Delta|:=\coprod_{F \in \Delta}|F|^{\circ}$ is a finite regular cell complex.

For a $\mathbb{Z}^{n}$-graded $k[Q]$-module $M$ and $\mathbf{a} \in \mathbb{Z}^{n}, M_{\mathbf{a}}$ denotes the degree a component of $M$. Let ${ }^{*}$ Mod be the category of $\mathbb{Z}^{n}$-graded $k[Q]$-modules. Here a morphism $f$ in ${ }^{*}$ Mod is a $k[Q]$-homomorphism $f: M \rightarrow N$ with $f\left(M_{\mathbf{a}}\right) \subset N_{\mathbf{a}}$ for all $\mathbf{a} \in \mathbb{Z}^{n}$.

We assign an order ideal $\Delta \subset L$ to the ideal

$$
I_{\Delta}:=\left(x^{\mathbf{a}} \mid \mathbf{a} \in Q \text { and } \operatorname{supp}(\mathbf{a}) \notin \Delta\right)
$$

of $k[Q]$. Set $k[\Delta]:=k[Q] / I_{\Delta}$. Clearly,

$$
k[\Delta]_{\mathbf{a}} \cong \begin{cases}k & \text { if } \mathbf{a} \in Q \text { and } \operatorname{supp}(\mathbf{a}) \in \Delta, \\ 0 & \text { otherwise }\end{cases}
$$

In particular, if $\Delta=L$ (resp. $\Delta=\emptyset$ ), then $I_{\Delta}=0$ (resp. $\left.I_{\Delta}=k[Q]\right)$ and $k[\Delta]=k[Q]$ (resp. $k[\Delta]=0)$. If $\Delta \neq \emptyset$ or $\{\{0\}\}$, then $\operatorname{dim} k[\Delta]=\operatorname{dim}|\Delta|+1$, where $\operatorname{dim}|\Delta|$ is the dimension as a cell complex. When $k[Q]$ is a polynomial ring, $k[\Delta]$ is nothing other than the Stanley-Reisner ring of a simplicial complex $\Delta$. (If $k[Q]$ is simplicial, $\Delta$ can be seen as a simplicial complex, and $|\Delta|=$ $\coprod_{F \in \Delta}|F|^{\circ}$ is homeomorphic to the geometric realization of $\Delta$ as a simplicial complex.)

We now recall the definition of squarefree $k[\Delta]$-modules.

Definition 2.1 ([Y1, Y2]). A $\mathbb{Z}^{n}$-graded $k[Q]$-module $M=\bigoplus_{\mathbf{a} \in \mathbb{Z}^{n}} M_{\mathbf{a}}$ is squarefree if the following two conditions are satisfied:

(1) $M$ is finitely generated and $Q$-graded (i.e., $M_{\mathbf{a}}=0$ for all $\mathbf{a} \notin Q$ ).

(2) The multiplication map $M_{\mathbf{a}} \ni y \mapsto x^{\mathbf{b}} y \in M_{\mathbf{a}+\mathbf{b}}$ is bijective for all $\mathbf{a}, \mathbf{b} \in Q$ with $\operatorname{supp}(\mathbf{a}+\mathbf{b})=\operatorname{supp}(\mathbf{a})$. 
The $\mathbb{Z}^{n}$-graded canonical module $K_{k[Q]}$ of $k[Q]$ is a squarefree module. In fact, $K_{k[Q]}$ is isomorphic to the ideal $\left(x^{\mathbf{a}} \mid \mathbf{a} \in Q\right.$ with $\left.\operatorname{supp}(\mathbf{a})=\mathbb{R}_{\geq 0} Q\right)$ of $k[Q]$. The quotient rings $k[\Delta]$ (in particular, $k[Q]$ itself) are also squarefree.

If $M$ is squarefree, then $M_{\mathbf{a}} \cong M_{\mathbf{b}}$ for all $\mathbf{a}, \mathbf{b} \in Q$ with $\operatorname{supp}(\mathbf{a})=\operatorname{supp}(\mathbf{b})$. In fact, $\operatorname{since} \operatorname{supp}(\mathbf{a})=\operatorname{supp}(\mathbf{a}+\mathbf{b})=\operatorname{supp}(\mathbf{b})$, we have $M_{\mathbf{a}} \cong M_{\mathbf{a}+\mathbf{b}} \cong M_{\mathbf{b}}$.

Denote the full subcategory of * Mod consisting of all squarefree $k[Q]$-modules by Sq. For $M \in{ }^{*}$ Mod and $\mathbf{a} \in \mathbb{Z}^{n}, M(\mathbf{a})$ denotes the shifted module of $M$ with $M(\mathbf{a})_{\mathbf{b}}=M_{\mathbf{a}+\mathbf{b}}$. If $M, N \in{ }^{*} \operatorname{Mod}$ and $M$ is finitely generated, then $\operatorname{Hom}_{k[Q]}(M, N)$ has the natural $\mathbb{Z}^{n}$-grading with

$$
\left[\operatorname{Hom}_{k[Q]}(M, N)\right]_{\mathbf{a}}=\operatorname{Hom}_{* \operatorname{Mod}}(M, N(\mathbf{a})) .
$$

Lemma 2.2 ([Y2, §4]). (1) Sq is a thick abelian subcategory of * $\mathrm{Mod}$ (i.e., closed under kernels, cokernels, and extensions in *Mod).

(2) $\mathrm{Sq}$ is an abelian category with enough projectives and injectives. An indecomposable projective (resp. injective) object in $\mathrm{Sq}$ is isomorphic to

$$
\begin{gathered}
J_{F}:=\left(x^{\mathbf{a}} \mid \mathbf{a} \in Q \text { with } \operatorname{supp}(\mathbf{a}) \supset F\right) \subset k[Q] \\
\left(\text { resp. } k[F]:=k[Q] /\left(x^{\mathbf{a}} \mid \mathbf{a} \in Q \text { with } \operatorname{supp}(\mathbf{a}) \not \subset F\right) \quad\right)
\end{gathered}
$$

for some $F \in L$. And both proj. $\operatorname{dim}_{\mathrm{Sq}} M$ and $\mathrm{inj} . \operatorname{dim}_{\mathrm{Sq}} M$ are at most $n$ for all $M \in \mathrm{Sq}$.

(3) The projective object $J_{F}$ is a Cohen-Macaulay $k[Q]$-module of dimension n. And

$\operatorname{Hom}_{k[Q]}\left(J_{F}, K_{k[Q]}\right) \cong\left(x^{\mathbf{a}} \mid \mathbf{a} \in Q\right.$ such that $\left.\operatorname{supp}(\mathbf{a}) \vee F=\mathbb{R}_{\geq 0} Q\right)$,

where $\operatorname{supp}(\mathbf{a}) \vee F \in L$ is the smallest face containing both $\operatorname{supp}(\mathbf{a})$ and $F$. In particular, $\operatorname{Hom}_{k[Q]}\left(J_{F}, K_{k[Q]}\right)$ is squarefree again.

For derived categories, we use the same notation as $[\mathrm{H}]$ (unless otherwise specified). In particular, for a module $M$ and an integer $i, M[i]$ means the complex $\cdots \rightarrow 0 \rightarrow M \rightarrow 0 \rightarrow \cdots$ with $M$ at the $(-i)^{\text {th }}$ place.

Lemma 2.3. We have the canonical category equivalence $D^{b}(\mathrm{Sq}) \cong D_{\mathrm{Sq}}^{b}\left({ }^{*} \mathrm{Mod}\right)$, and $D^{b}(\mathrm{Sq})$ can be seen as a full subcategory of $D^{b}\left({ }^{*} \mathrm{Mod}\right)$.

Proof. Let ${ }^{*} \operatorname{Mod}_{Q}$ be the full subcategory of ${ }^{*} \operatorname{Mod}$ consisting of finitely generated $Q$-graded modules. Then ${ }^{*} \operatorname{Mod}_{Q}$ is a thick abelian subcategory of ${ }^{*}$ Mod. Moreover, ${ }^{*} \operatorname{Mod}_{Q}$ has enough projectives, and projective objects $k[Q](-\mathbf{a})$ with $\mathbf{a} \in Q$ are also projective in ${ }^{*} \operatorname{Mod}$. Thus $D^{*}{ }^{b} \operatorname{Mod}_{Q}\left({ }^{*} \operatorname{Mod}\right) \cong D^{b}\left({ }^{*} \operatorname{Mod}_{Q}\right)$ and $D^{b}{ }^{*} \operatorname{Mod}_{Q}\left({ }^{*} \mathrm{Mod}\right)$ is a full subcategory of $D^{b}\left({ }^{*} \mathrm{Mod}\right)$. On the other hand, Sq is a thick abelian subcategory of ${ }^{*} \operatorname{Mod}_{Q}$, and an injective object $k[F]$ of Sq is also injective in ${ }^{*} \operatorname{Mod}_{Q}$ by $[\mathrm{M}, \operatorname{Remark} 2.5]$. So $D^{b}(\mathrm{Sq}) \cong D_{\mathrm{Sq}}^{b}\left({ }^{*} \operatorname{Mod}_{Q}\right)$, and $D_{\mathrm{Sq}}^{b}\left({ }^{*} \operatorname{Mod}_{Q}\right)$ is a full subcategory of $D^{b}\left({ }^{*} \operatorname{Mod}_{Q}\right)$, which can be seen as a full subcategory of $D^{b}\left({ }^{*} \mathrm{Mod}\right)$. 
Next we will study $R \operatorname{Hom}_{k[Q]}\left(M^{\bullet}, K_{k[Q]}\right)$ for a complex $M^{\bullet} \in D^{b}\left({ }^{*} \mathrm{Mod}\right)$. Here $R \operatorname{Hom}_{k[Q]}\left(M^{\bullet}, K_{k[Q]}\right)$ is the " $R$ Hom" as complexes of (non-graded) $k[Q]$ modules. But if each $H^{i}\left(M^{\bullet}\right)$ is finitely generated, $R \operatorname{Hom}_{k[Q]}\left(M^{\bullet}, K_{k[Q]}\right)$ has a natural $\mathbb{Z}^{n}$-grading, and defines an object in $D^{b}\left({ }^{*} \mathrm{Mod}\right)$. In fact, if $P^{\bullet}$ is a $\mathbb{Z}^{n}$-graded finite free resolution of $M^{\bullet}$, then

$$
R \operatorname{Hom}_{k[Q]}\left(M^{\bullet}, K_{k[Q]}\right) \cong \operatorname{Hom}_{k[Q]}^{\bullet}\left(P^{\bullet}, K_{k[Q]}\right)
$$

and each $\operatorname{Hom}_{k[Q]}^{i}\left(P^{\bullet}, K_{k[Q]}\right)\left(=\operatorname{Hom}_{k[Q]}\left(P^{-i}, K_{k[Q]}\right)\right)$ has the $\mathbb{Z}^{n}$-grading. We can also define $R \operatorname{Hom}_{k[Q]}\left(M^{\bullet}, K_{k[Q]}\right)$ using a $\mathbb{Z}^{n}$-graded injective resolution $I^{\bullet} \in$ $D^{b}\left({ }^{*} \mathrm{Mod}\right)$ of $K_{k[Q]}$, but we get the same $\mathbb{Z}^{n}$-grading.

Lemma 2.4. If $M^{\bullet} \in D_{\mathrm{Sq}}^{b}\left({ }^{*} \operatorname{Mod}\right)$, then $R \operatorname{Hom}_{k[Q]}\left(M^{\bullet}, K_{k[Q]}\right)$ is in $D_{\mathrm{Sq}}^{b}\left({ }^{*} \operatorname{Mod}\right)$ too. That is, $\operatorname{Ext}_{k[Q]}^{i}\left(M^{\bullet}, K_{k[Q]}\right)$ is squarefree for all $i$.

Proof. By Lemma 2.3, we may assume that $M^{\bullet} \in D^{b}(\mathrm{Sq})$. Then we have a projective resolution $P^{\bullet} \in D^{b}(\mathrm{Sq})$ of $M^{\bullet}$. By Lemma $2.2(3), \operatorname{Ext}_{k[Q]}^{i}\left(P^{j}, K_{k[Q]}\right)=0$ for all $i \neq 0$ and all $j$. Hence we have

$$
R \operatorname{Hom}_{k[Q]}\left(M^{\bullet}, K_{k[Q]}\right) \cong \operatorname{Hom}_{k[Q]}^{\bullet}\left(P^{\bullet}, K_{k[Q]}\right) .
$$

But each $\operatorname{Hom}_{k[Q]}\left(P^{j}, K_{k[Q]}\right)$ is squarefree by Lemma $2.2(3)$.

Take some $\mathbf{a}(F) \in Q \cap \operatorname{rel}-i n t(F)$ for each $F \in L$. For a squarefree module $M$, set $M_{F}:=M_{\mathrm{a}(F)}$. If $F, G \in L$ and $G \supset F$, [Y2, Theorem 3.3] gives a $k$-linear $\operatorname{map} \varphi_{G, F}^{M}: M_{F} \rightarrow M_{G}$. These maps satisfy $\varphi_{F, F}^{M}=\operatorname{Id}$ and $\varphi_{G, F}^{M} \circ \varphi_{F, E}^{M}=\varphi_{G, E}^{M}$ for all $G \supset F \supset E$. For $F \in L$, we define the complex $C_{F}^{\bullet}(M): 0 \rightarrow C_{F}^{0} \rightarrow$ $C_{F}^{1} \rightarrow \cdots \rightarrow C_{F}^{n} \rightarrow 0$ of $k$-vector spaces by

$$
C_{F}^{i}=\bigoplus_{\substack{G \in L, G \supset F \\ \operatorname{dim} G=i}} M_{G}
$$

and the differential

$$
d: C_{F}^{i} \supset M_{G} \ni y \longmapsto \sum_{\substack{G^{\prime} \in L, G^{\prime} \supset G \\ \operatorname{dim} G^{\prime}=i+1}} \varepsilon\left(G^{\prime}, G\right) \cdot \varphi_{G^{\prime}, G}^{M}(y) \in \bigoplus_{\substack{G^{\prime} \in L, G^{\prime} \supset G \\ \operatorname{dim} G^{\prime}=i+1}} M_{G^{\prime}} \subset C_{F}^{i+1}
$$

Here $\varepsilon$ is an incidence function on the cell complex $B=\coprod_{F \in L}|F|^{\circ}$. The complex $C_{F}^{\bullet}(M)$ does not depend on the particular choice of $\mathbf{a}(F)$ 's up to isomorphism. By the computation of a Čech complex with supports in $\mathfrak{m}$, we have the following.

Lemma 2.5 ([Y2, Theorem 3.10]). Let the notation be as above. If $\mathbf{a} \notin Q$, then $\left[H_{\mathfrak{m}}^{i}(M)\right]_{-\mathbf{a}}=0$. If $\mathbf{a} \in Q$ and $\operatorname{supp}(\mathbf{a})=F$, then $\left[H_{\mathfrak{m}}^{i}(M)\right]_{-\mathbf{a}} \cong H^{i}\left(C_{F}^{\bullet}(M)\right)$. 


\section{Sheaves associated with squarefree modules}

We keep the same notation as above. For a squarefree module $M$, set

$$
\operatorname{Spé}(M):=\coprod_{F \in L}|F|^{\circ} \times M_{F} .
$$

Let $\pi: \operatorname{Spé}(M) \rightarrow B$ be the projection map which sends $(p, m) \in|F|^{\circ} \times M_{F} \subset$ Spé $(M)$ to $p \in|F|^{\circ} \subset B$. For an open subset $U \subset B$ and a map $s: U \rightarrow \operatorname{Spé}(M)$, we will consider the following conditions:

(*) $\pi \circ s=\operatorname{Id}_{U}$ and $s_{q}=\varphi_{G, F}^{M}\left(s_{p}\right)$ for all $p, q \in U$ such that $F:=\operatorname{supp}(p)$ is contained in $G:=\operatorname{supp}(q)$. Here $s_{p}$ (resp. $s_{q}$ ) is the element of $M_{F}$ (resp. $\left.M_{G}\right)$ with $s(p)=\left(p, s_{p}\right)$ (resp. $\left.s(q)=\left(q, s_{q}\right)\right)$.

(**) There is an open covering $U=\bigcup_{\lambda \in \Lambda} U_{\lambda}$ such that the restriction of $s$ to $U_{\lambda}$ satisfies $(*)$ for all $\lambda \in \Lambda$.

Now we define the $k$-sheaf associated to $M$ on $B$, denoted by $M^{+}$, as follows. The sections $M^{+}(U)$ of $M^{+}$over an open set $U$ is

$$
\{s \mid s: U \rightarrow \operatorname{Spé}(M) \text { is a map satisfying }(* *)\}
$$

and the restriction map $M^{+}(U) \rightarrow M^{+}(V)$ is the natural one. (That $M^{+}$is actually a sheaf is obvious.)

We say an open set $U$ of $B$ is neat with respect to a face $F \in L$, if $U$ itself and $U \cap|G|^{\circ}$ are connected for all $G \in L$ with $G \supset F$, and $q \in U$ implies $\operatorname{supp}(q) \supset F$. For example, the open set $U_{F}:=\coprod_{G \supset F}|G|^{\circ}$ is neat with respect to $F$. For $x \in|F|^{\circ}$ and sufficiently small $\varepsilon>0, U_{\varepsilon}(x):=\{y \in B \mid d(x, y)<\varepsilon\}$ is also neat with respect to $F$, where $d(-,-)$ stands for the usual metric of $\mathbb{R}^{n}(\supset B)$. We can easily check the following.

(i) Assume that $U \cap|F|^{\circ}$ is connected, and let $s \in M^{+}(U)$ be a section. Then there is some $y \in M_{F}$ such that $s(p)=(p, y)$ for all $p \in U \cap|F|^{\circ}$.

(ii) Assume that $U$ is neat with respect to $F$. For any $y \in M_{F}$, the map $s_{y}: U \rightarrow \operatorname{Spé}(M)$ defined by $\left(U \cap|G|^{\circ}\right) \ni p \mapsto\left(p, \varphi_{G, F}(y)\right)$ satisfies $(*)$. In particular, $s_{y} \in M^{+}(U)$.

(iii) If $U$ is neat with respect to $F$, any section $s \in M^{+}(U)$ coincides with $s_{y}$ of (ii) for some $y \in M_{F}$.

Hence, if $U$ is neat with respect to $F$, then $M^{+}(U) \cong M_{F}$. Note that the set of neat open sets is an open base of $B$. Thus, for a point $p \in|F|^{\circ}$, the stalk $\left(M^{+}\right)_{p}$ of $M^{+}$at $p$ is isomorphic to $M_{F}$. So Spé $(M)$ is the etale space of the sheaf $M^{+}$.

Let $\Psi \subset L$ be an order filter of the poset $L$, that is, $F \in \Psi, G \in L$, and $G \supset F$ imply $G \in \Psi$. Then $U_{\Psi}:=\coprod_{F \in \Psi}|F|^{\circ}$ is an open subset of $B$. If $M$ is a squarefree module, then the submodule

$$
M_{\Psi}:=\bigoplus_{\mathbf{a} \in Q, \operatorname{supp}_{+}(\mathbf{a}) \in \Psi} M_{\mathbf{a}}
$$

is also squarefree. Moreover, we have the following. 
Lemma 3.1. The sheaf $\left(M_{\Psi}\right)^{+}$is isomorphic to $j ! j^{*} M^{+}$, where $j: U_{\Psi} \rightarrow B$ is the embedding map.

Proof. Straightforward.

Example 3.2. (1) Let $\Delta \subset L$ be an order ideal, and $j$ the embedding map from the closed subset $|\Delta|=\coprod_{F \in \Delta}|F|$ to $B$. Then the sheaf $k[\Delta]^{+}$is isomorphic to $j_{*} \underline{k}_{|\Delta|}$, where $\underline{k}_{|\Delta|}$ is the constant sheaf on $|\Delta|$.

(2) Let $J_{F}$ be the projective object in Sq associated with a face $F \in L$. Then the sheaf $\left(J_{F}\right)^{+}$is isomorphic to $j ! \underline{k}_{U_{F}}$, where $j$ is the embedding map from the open set $U_{F}=\coprod_{G \in L, G \supset F}|G|^{\circ}$ to $B$. Note that

$$
U_{F} \cong \begin{cases}\mathbb{R}^{n-1}, & \text { if } F=\mathbb{R}_{\geq 0} Q, \\ \mathbb{R}_{+}^{n-1}:=\left\{\left(y_{1}, \ldots, y_{n-1}\right) \in \mathbb{R}^{n-1} \mid y_{n-1} \geq 0\right\}, & \text { if } F \neq \mathbb{R}_{\geq 0} Q,\{0\}, \\ B^{n-1}:=\left\{\left(y_{1}, \ldots, y_{n-1}\right) \in \mathbb{R}^{n-1} \mid \sum_{i=1}^{n-1} y_{i}^{2} \leq 1\right\}, & \text { if } F=\{0\} .\end{cases}
$$

(3) Let $\Delta, \Sigma \subset L$ be order ideals with $\Delta \supset \Sigma$. We have $I_{\Delta} \subset I_{\Sigma}$. Set $I_{\Delta / \Sigma}:=I_{\Sigma} / I_{\Delta}$ (see [Sta, III.7]). If $\Sigma=\emptyset($ resp. $\Delta=L)$, then $I_{\Delta / \Sigma}=k[\Delta]$ (resp. $I_{\Delta / \Sigma}=I_{\Sigma}$ ). It is easy to see that $I_{\Delta / \Sigma}$ is a squarefree module with $\left(I_{\Delta / \Sigma}\right)^{+} \cong j_{!} \underline{k}_{|\Delta|-|\Sigma|}$, where $j$ is the embedding map from the locally closed subset $|\Delta|-|\Sigma|$ to $B$.

For a topological space $X, \operatorname{Sh}(X)$ denotes the category of $k$-sheaves on $X$ (i.e., the category of $\underline{k}_{X}$-modules).

If $M$ is a squarefree module, $M_{>0}$ denotes the submodule $\bigoplus_{\mathbf{a} \in Q \backslash\{0\}} M_{\mathbf{a}}$ of $M$. Then $M_{>0}$ is squarefree again, and $M^{+} \cong\left(M_{>0}\right)^{+}$. For a complex $0 \rightarrow$ $L \rightarrow M \rightarrow N \rightarrow 0$ of squarefree modules, the complex of sheaves $0 \rightarrow L^{+} \rightarrow$ $M^{+} \rightarrow N^{+} \rightarrow 0$ is exact if and only if $0 \rightarrow L_{F} \rightarrow M_{F} \rightarrow N_{F} \rightarrow 0$ is exact for all $\{0\} \neq F \in L$. Hence the functor $(-)^{+}: \mathrm{Sq} \rightarrow \mathrm{Sh}(B)$ is exact. But this functor is neither full nor faithful. The degree 0 component $M_{0}$ causes this problem. Let $\mathrm{Sq}_{+}$be the full subcategory of Sq consisting of all $M$ with $M_{0}=0$. It is easy to see that the functor $(-)^{+}: \mathrm{Sq}_{+} \rightarrow \mathrm{Sh}(B)$ is fully faithful.

Theorem 3.3. If $M$ is a squarefree $k[Q]$-module, we have an isomorphism

$$
H^{i}\left(B, M^{+}\right) \cong\left[H_{\mathfrak{m}}^{i+1}(M)\right]_{0} \text { for all } i \geq 1,
$$

and an exact sequence

$$
0 \rightarrow\left[H_{\mathfrak{m}}^{0}(M)\right]_{0} \rightarrow M_{0} \rightarrow H^{0}\left(B, M^{+}\right) \rightarrow\left[H_{\mathfrak{m}}^{1}(M)\right]_{0} \rightarrow 0
$$

In particular, if $M \in \mathrm{Sq}_{+}$, then $H^{i}\left(B, M^{+}\right) \cong\left[H_{\mathfrak{m}}^{i+1}(M)\right]_{0}$ for all $i \geq 0$.

Proof. As usual, let $\Gamma_{\mathfrak{m}}:{ }^{*} \operatorname{Mod} \rightarrow{ }^{*} \operatorname{Mod}$ be the functor defined by $\Gamma_{\mathfrak{m}}(N):=$ $\left\{y \in N \mid \mathfrak{m}^{l} y=0\right.$ for $\left.l \gg 0\right\}$, and $\Gamma(B,-): \operatorname{Sh}(B) \rightarrow$ vect $_{k}$ the global sections functor.

Let $I^{\bullet}$ (resp. $\check{I}^{\bullet}$ ) be a minimal injective resolution of $M$ in Sq (resp. in ${ }^{*}$ Mod), and consider the exact sequence

$$
0 \rightarrow \Gamma_{\mathfrak{m}}\left(I^{\bullet}\right) \rightarrow I^{\bullet} \rightarrow I^{\bullet} / \Gamma_{\mathfrak{m}}\left(I^{\bullet}\right) \rightarrow 0
$$


of cochain complexes. Put $J^{\bullet}:=I^{\bullet} / \Gamma_{\mathfrak{m}}\left(I^{\bullet}\right)$. Each component of $J^{\bullet}$ is a direct sum of copies of $k[F]$ for various $\{0\} \neq F \in L$. Since $k[F]^{+}$is the constant sheaf on $|F|$ which is homeomorphic to a closed ball, we have $H^{i}\left(B, k[F]^{+}\right)=$ $H^{i}(|F| ; k)=0$ for all $i \geq 1$. Hence $\left(J^{\bullet}\right)^{+}\left(\cong\left(I^{\bullet}\right)^{+}\right)$gives a $\Gamma(B,-)$-acyclic resolution of $M^{+}$. It is easy to see that $\left[J^{\bullet}\right]_{0} \cong \Gamma\left(B,\left(J^{\bullet}\right)^{+}\right)$. By [M, Theorem 2.4], $I^{\bullet}$ coincides with the $Q$-graded part $\bigoplus_{\mathbf{a} \in Q}\left[\check{I}^{\bullet}\right]_{\mathbf{a}}$ of $I^{\bullet}$. Thus we have $\left[H^{i}\left(\Gamma_{\mathfrak{m}}\left(I^{\bullet}\right)\right)\right]_{0}=\left[H^{i}\left(\Gamma_{\mathfrak{m}}\left(\check{I}^{\bullet}\right)\right)\right]_{0}=\left[H_{\mathfrak{m}}^{i}(M)\right]_{0}$. So the first and the second assertions follow from $(3.2)$, since $\left[H^{0}\left(I^{\bullet}\right)\right]_{0} \cong M_{0}$ and $H^{i}\left(I^{\bullet}\right)=0$ for all $i \geq 1$.

To prove the last isomorphism, we may assume that $i=0$. But the isomorphism follows from the exact sequence $(3.1)$, since $H_{\mathfrak{m}}^{0}(M)=M_{0}=0$ in this case.

Remark 3.4. Let $M$ be a finitely generated $\mathbb{Z}$-graded module over $S=k\left[x_{1}, \ldots, x_{n}\right]$. Then we have an algebraic coherent sheaf $\tilde{M}$ on $\mathbb{P}^{n-1}=\operatorname{Proj}(S)$. Like our functor $(-)^{+}$, if $\operatorname{dim}_{k} M<\infty$, then $\tilde{M}=0$. Moreover, it is well-known that $H^{i}\left(\mathbb{P}^{n-1}, \tilde{M}\right) \cong\left[H_{\mathfrak{m}}^{i+1}(M)\right]_{0}$ for all $i \geq 1$, and

$$
0 \rightarrow\left[H_{\mathfrak{m}}^{0}(M)\right]_{0} \rightarrow M_{0} \rightarrow H^{0}\left(\mathbb{P}^{n-1}, \tilde{M}\right) \rightarrow\left[H_{\mathfrak{m}}^{1}(M)\right]_{0} \rightarrow 0 \quad \text { (exact), }
$$

(see, for example, [SV, p.38]). So Theorem 3.3 gives an analogy between Proj and our $(-)^{+}$.

Recall that we chose $\mathbf{a}(F) \in Q \cap F$ for each $F \in L$ in the previous section. By the graded local duality, the $\mathbb{Z}^{n}$-graded $k$-dual of $H_{\mathfrak{m}}^{i}(M)$ is isomorphic to the squarefree module $\operatorname{Ext}_{k[Q]}^{n-i}\left(M, K_{k[Q]}\right)$. So to determine the $\mathbb{Z}^{n}$-graded Hilbert function of $H_{\mathfrak{m}}^{i}(M)$, it suffices to know $\left[H_{\mathfrak{m}}^{i}(M)\right]_{-\mathbf{a}(F)}$ for each $F$. Since Theorem 3.3 deals with the case when $F=\{0\}$ (i.e., $\mathbf{a}(F)=0$ ), we may assume that $F \neq\{0\}$.

Theorem 3.5. Let $M$ be a squarefree $k[Q]$-module, and $j$ the embedding map from the open set $U_{F}=\coprod_{G \supset F}|G|^{\circ}$ to $B$. If $F \neq\{0\}$, we have

$$
H_{c}^{i}\left(U_{F}, j^{*} M^{+}\right) \cong\left[H_{\mathfrak{m}}^{i+1}(M)\right]_{-\mathbf{a}(F)} \text { for all } i \geq 0,
$$

where $H_{c}^{i}(-)$ stands for the cohomology with the compact support.

Proof. Let $\Psi:=\{G \in L \mid G \supset F\}$ be the order filter of $L$. Under the same notation as Lemma 3.1, we have $U_{\psi}=U_{F}$. We have the following.

$$
\begin{aligned}
{\left[H_{\mathfrak{m}}^{i+1}(M)\right]_{-\mathbf{a}(F)} } & \cong H^{i+1}\left(C_{F}^{\bullet}(M)\right) \quad(\text { by Lemma } 2.5) \\
& \cong H^{i+1}\left(C_{\{0\}}^{\bullet}\left(M_{\Psi}\right)\right) \\
& \cong\left[H_{\mathfrak{m}}^{i+1}\left(M_{\Psi}\right)\right]_{0} \quad(\text { by Lemma } 2.5) \\
& \cong H^{i}\left(B,\left(M_{\Psi}\right)^{+}\right) \quad\left(\text { by Theorem } 3.3 . \text { Note that } M_{\Psi} \in \mathrm{Sq}_{+}\right) \\
& \cong H^{i}\left(B, j ! j^{*} M^{+}\right) \quad(\text { by Lemma } 3.1) \\
& \left.\cong H_{c}^{i}\left(U_{F}, j^{*} M^{+}\right) \quad(\text { by [I, III, Corollary } 7.3]\right)
\end{aligned}
$$


Remark 3.6. When $k[Q]$ is a polynomial ring $k\left[\mathbb{N}^{n}\right]=k\left[x_{1}, \ldots, x_{n}\right]$, Theorems 3.3 and 3.5 generalize a well-known formula of Hochster ([BH, Theorem 5.3.8], see also [BH, Lemma 5.4.5]). This formula states that $\left[H_{\mathfrak{m}}^{i+1}(k[\Delta])\right]_{0} \cong$ $\tilde{H}_{i}(|\Delta| ; k)$ for all $i \geq 0$, where the right hand side is the $i$ th reduced homology group of $|\Delta|$. On the other hand, Theorem 3.3 states that $\left[H_{\mathfrak{m}}^{i+1}(k[\Delta])\right]_{0}=$ $H^{i}\left(B, k[\Delta]^{+}\right)$for all $i \geq 1$ and $H^{0}\left(B, k[\Delta]^{+}\right)=\left[H_{\mathfrak{m}}^{1}(k[\Delta])\right]_{0} \oplus k[\Delta]_{0} \cong\left[H_{\mathfrak{m}}^{1}(k[\Delta])\right]_{0}$ $\oplus k$. But $H^{i}\left(B, k[\Delta]^{+}\right)=H^{i}\left(B, j_{*} \underline{k}_{|\Delta|}\right)=H^{i}(|\Delta| ; k)=\tilde{H}_{i}(|\Delta| ; k)$ for all $i \geq 1$, and $H^{0}\left(B, k[\Delta]^{+}\right)=H_{0}(|\Delta| ; k)=\tilde{H}_{0}(|\Delta| ; k) \oplus k$. So Theorem 3.3 coincides with Hochster's formula. If $0 \neq \mathbf{a} \in \mathbb{N}^{n}$ and $\operatorname{supp}(\mathbf{a})=F$, Hochster's formula states that $\left[H_{\mathfrak{m}}^{i+1}(k[\Delta])\right]_{-\mathbf{a}}=H_{i}(|\Delta|,|\Delta|-\{p\} ; k)$ for a point $p \in|F|^{\circ}$. Set $u_{F}:=U_{F} \cap|\Delta|$. For $p \in|F|^{\circ}, u_{F}$ is a cone neighbourhood of $p$ and $|\Delta|-u_{F}$ is a deformation retract of $|\Delta|-\{p\}$. Hence we have

$$
\begin{aligned}
{\left[H_{\mathfrak{m}}^{i+1}(M)\right]_{-\mathbf{a}} } & \cong H_{c}^{i}\left(U_{F}, j^{*} k[\Delta]^{+}\right) \quad \text { (by Theorem 3.5) } \\
& \cong H_{c}^{i}\left(u_{F}, \underline{k}_{u_{F}}\right) \\
& \cong H^{i}\left(|\Delta|,|\Delta|-u_{F} ; k\right) \quad(\text { see }[\text { I, IV. Definition 8.1]) } \\
& \cong H^{i}(|\Delta|,|\Delta|-\{p\} ; k) .
\end{aligned}
$$

So Theorem 3.5 and Hochster's formula also coincide.

\section{Relation to Poincaré-Verdier Duality}

Since the functor $(-)^{+}: \mathrm{Sq} \rightarrow \mathrm{Sh}(B)$ is exact, it can be extended to the functor $(-)^{+}: D^{b}(\mathrm{Sq}) \rightarrow D^{b}(\mathrm{Sh}(B))$. If $M^{\bullet} \in D^{b}(\mathrm{Sq})$, we have $R \operatorname{Hom}_{k[Q]}\left(M^{\bullet}, K_{k[Q]}\right)$ $\in D_{\mathrm{Sq}}^{b}\left({ }^{*} \mathrm{Mod}\right)$ by Lemma 2.4. So there is a bounded complex $N^{\bullet}$ of squarefree modules such that $N^{\bullet} \cong R \operatorname{Hom}_{k[Q]}\left(M^{\bullet}, K_{k[Q]}\right)$ in $D^{b}\left({ }^{*} \operatorname{Mod}\right)$. We denote $\left(N^{\bullet}\right)^{+} \in D^{b}(\operatorname{Sh}(B))$ by $R \operatorname{Hom}_{k[Q]}\left(M^{\bullet}, K_{k[Q]}\right)^{+} . R \operatorname{Hom}_{k[Q]}\left(M^{\bullet}, K_{k[Q]}\right)^{+}$does not depend on the particular choice of $N^{\bullet}$ up to isomorphism in $D^{b}(\operatorname{Sh}(B))$, of course.

For a locally compact topological space $X$ of finite dimension (e.g., a locally closed subset of $B), \mathcal{D}_{X}^{\bullet}$ denotes a dualizing complex of $X$ with the coefficients in $k$ (see [I, V. $\S 2]$ ). In this paper, we frequently use the isomorphism $\mathcal{D}_{Y}^{\bullet} \cong$ $j^{!} \mathcal{D}_{X}^{\bullet}$ for the embedding map $j$ from a locally closed subset $Y$ to $X$ (see [I, V. Theorem 5.6]). If $X$ is a manifold (with or without boundary), we have the orientation sheaf or $_{X}$ of $X$ with the coefficients in $k$. In this case, we have $\mathcal{D}_{X}^{\bullet} \cong \operatorname{or}_{X}[\operatorname{dim} X]($ see $[\mathrm{I}, \mathrm{V} . \S 3])$.

Lemma 4.1. With the above notation, we have the following.

(1) or $_{B} \cong\left(K_{k[Q]}\right)^{+}$.

(2) Let $J_{F}$ be the projective object in $\mathrm{Sq}$ associated with a face $F \in L$. Then $R \mathcal{H o m}_{\mathrm{Sh}(B)}\left(\left(J_{F}\right)^{+}\right.$, or $\left._{B}\right) \cong \operatorname{Hom}_{k[Q]}\left(J_{F}, K_{k[Q]}\right)^{+}$.

(3) If $M^{\bullet} \in D^{b}(\mathrm{Sq})$, we have an isomorphism

$$
R \mathcal{H o m}_{\mathrm{Sh}(B)}\left(\left(M^{\bullet}\right)^{+}, \text {or }_{B}\right) \cong R \operatorname{Hom}_{k[Q]}\left(M^{\bullet}, K_{k[Q]}\right)^{+}
$$

in $D^{b}(\operatorname{Sh}(B))$. 
Proof. (1) Let $\underline{k}_{B^{\circ}}$ be the constant sheaf on the relative interior $B^{\circ}$ of $B$. If $j: B^{\circ} \rightarrow B$ is the embedding map, then ${ }^{\circ} r_{B} \cong j ! \underline{k}_{B^{\circ}}$ by [I, VI. Proposition 3.3]. On the other hand, $\left(K_{k[Q]}\right)^{+} \cong j ! \underline{k}_{B^{\circ}}$ as we have seen in Example 3.2.

(2) Recall that if $U$ is an open set with the embedding map $j: U \rightarrow B$ and $\mathcal{I}$ is an injective object in $\operatorname{Sh}(B)$, then $j^{*} \mathcal{I}\left(=j^{!} \mathcal{I}\right)$ is injective in $\operatorname{Sh}(U)$. So $\mathcal{E} x t_{\mathrm{Sh}(B)}^{i}\left(\left(J_{F}\right)^{+}\right.$, or $\left._{B}\right)$ is the sheaf associated to the presheaf which sends an open set $U$ to $\operatorname{Ext}_{\operatorname{Sh}(U)}^{i}\left(j^{*}\left(J_{F}\right)^{+}, j^{*} o r_{B}\right)$. Note that $j^{*} o r_{B} \cong o r_{U}$. By the PoincaréVerdier duality ([I, V. 2.1]), we have

$$
\operatorname{Ext}_{\operatorname{Sh}(U)}^{i}\left(j^{*}\left(J_{F}\right)^{+}, j^{*} o r_{B}\right) \cong H_{c}^{n-1-i}\left(U, j^{*}\left(J_{F}\right)^{+}\right)^{\vee},
$$

where $(-)^{\vee}$ means the dual $k$-vector space. For any open neighbourhood $V$ of $p$, there is an open set $U$ with $p \in U \subset V$ such that $U \cap U_{F} \cong \mathbb{R}^{n-1}$ or $\mathbb{R}_{+}^{n-1}$. Then $H_{c}^{i}\left(U, j^{*}\left(J_{F}\right)^{+}\right) \cong H_{c}^{i}\left(U \cap U_{F} ; k\right)=0$ for all $i \neq n-1$. Thus $\mathcal{E} x t_{\mathrm{Sh}(B)}^{i}\left(\left(J_{F}\right)^{+}\right.$, or $\left._{B}\right)=0$ for all $i \neq 0$. Hence we have $R \mathcal{H} \operatorname{Hom}_{\mathrm{Sh}(B)}\left(\left(J_{F}\right)^{+}\right.$, or $\left._{B}\right) \cong$ $\mathcal{H o m}_{\mathrm{Sh}(B)}\left(\left(J_{F}\right)^{+}\right.$, or $\left._{B}\right)$.

Recall that $\left(J_{F}\right)^{+}$is the constant sheaf on $U_{F}$ and $o r_{B}$ is the constant sheaf on $B^{\circ}$. For a point $p \in B$, the stalk $\mathcal{H o m}_{\mathrm{Sh}(B)}\left(\left(J_{F}\right)^{+}\right.$, or $\left._{B}\right) p$ at $p$ is nonzero (equivalently, $\mathcal{H o m}_{\mathrm{Sh}(B)}\left(\left(J_{F}\right)^{+}, o r_{B}\right) p=k$ ) if and only if there is an open neighbourhood $U_{p}$ of $p$ such that $U_{p} \cap U_{F} \subset B^{\circ}$. With the same notation as Lemma 2.2 (3), the latter condition is equivalent to the condition that $\operatorname{supp}(p) \vee F=\mathbb{R}_{\geq 0} Q$. So the assertion follows from Lemma $2.2(3)$.

(3) Let $P^{\bullet}$ be a projective resolution of $M^{\bullet}$ in Sq, that is, there is a quasi isomorphism $P^{\bullet} \rightarrow M^{\bullet}$ and each $P^{i}$ is a direct sum of copies of $J_{F}$ for various $F$. By (2), we can compute $R \mathcal{H} \operatorname{Hom}_{S h(B)}\left(\left(M^{\bullet}\right)^{+}, o r_{B}\right)$ by $\left(P^{\bullet}\right)^{+}$. So we have

$$
\begin{aligned}
R \mathcal{H o m}_{\mathrm{Sh}(B)}\left(\left(M^{\bullet}\right)^{+}, \text {or }_{B}\right) & \cong \mathcal{H o m}_{\mathrm{Sh}(B)}^{\bullet}\left(\left(P^{\bullet}\right)^{+}, \text {or }_{B}\right) \\
& \cong \operatorname{Hom}_{k[Q]}^{\bullet}\left(P^{\bullet}, K_{k[Q]}\right)^{+} \\
& \cong R \operatorname{Hom}_{k[Q]}\left(M^{\bullet}, K_{k[Q]}\right)^{+}
\end{aligned}
$$

The normalized $\mathbb{Z}^{n}$-graded dualizing complex of $k[Q]$ is a $\mathbb{Z}^{n}$-graded injective resolution of $K_{k[Q]}[n]$. But, in this paper, we will consider a $\mathbb{Z}^{n}$-graded injective resolution of $K_{k[Q]}[n-1]$, which is a non-normalized dualizing complex. The reason why we use this convention is that $k[Q]$ represents the $(n-1)$-dimensional polytope $B$ in our context.

Let $\omega_{k[Q]}^{\bullet}$ be the $Q$-graded part of a minimal $\mathbb{Z}^{n}$-graded injective resolution of $K_{k[Q]}[n-1]$. The complex $\omega_{k[Q]}^{\bullet}$, which is a minimal injective resolution of $K_{k[Q]}[n-1]$ in $\mathrm{Sq}$, is of the form

$$
\begin{aligned}
\omega_{k[Q]}^{\bullet}: 0 \longrightarrow \omega^{-n+1} & \longrightarrow \omega^{-n+2} \longrightarrow \cdots \longrightarrow \omega^{1} \longrightarrow 0, \\
\omega^{i}= & \bigoplus_{\substack{F \in L \\
F=-i+1}} k[F],
\end{aligned}
$$


and the differential is composed of the maps $\varepsilon(F, G) \cdot$ nat $: k[F] \rightarrow k[G]$ for all $G \in L$ with $\operatorname{dim} G=\operatorname{dim} F-1$, where $\varepsilon$ is the incidence function on the cell complex $B=\coprod_{F \in L}|F|^{\circ}$ and nat $: k[F] \rightarrow k[G]$ is the natural surjection.

For an order ideal $\Delta \subset L$, set $\omega_{k[\Delta]}^{\bullet}:=\operatorname{Hom}_{k[Q]}\left(k[\Delta], \omega_{k[Q]}^{\bullet}\right)$. This is a complex of squarefree $k[\Delta]$-modules with

$$
\omega_{k[\Delta]}^{i}=\bigoplus_{\substack{F \in \Delta \\ \operatorname{dim} F=-i+1}} k[F] .
$$

Note that $\omega_{k[\Delta]}^{\bullet}$ is isomorphic to a non-normalized $\mathbb{Z}^{n}$-graded dualizing complex of $k[\Delta]$ in the derived category of $\mathbb{Z}^{n}$-graded $k[\Delta]$-modules.

Let $\mathrm{Sq}(\Delta)$ be the full subcategory of Sq consisting of $k[\Delta]$-modules, that is, $M \in \mathrm{Sq}(\Delta)$ if and only if $M$ is a squarefree $k[Q]$-module whose annihilator $\operatorname{Ann}(M)$ contains $I_{\Delta}$. The category $\operatorname{Sq}(\Delta)$ is a thick abelian subcategory of Sq, and $\operatorname{Sq}(\Delta)$ has enough injectives, and an indecomposable injective object is of the form $k[F]$ for some $F \in \Delta$ (c.f. [RWY]), which is also injective in Sq. Thus $D^{b}(\mathrm{Sq}(\Delta)) \cong D_{\mathrm{Sq}(\Delta)}^{b}(\mathrm{Sq}) \cong D_{\mathrm{Sq}(\Delta)}^{b}\left({ }^{*} \mathrm{Mod}\right)$, and $D^{b}(\mathrm{Sq}(\Delta))$ can be viewed as a full subcategory of $D^{b}\left({ }^{*} \mathrm{Mod}\right)$.

If $M^{\bullet} \in D^{b}(\mathrm{Sq}(\Delta))$, we have $R \operatorname{Hom}_{k[Q]}\left(M \bullet K_{k[Q]}[n-1]\right) \cong R \operatorname{Hom}_{k[\Delta]}\left(M \bullet \omega_{k[\Delta]}^{\bullet}\right)$ in $D^{b}\left({ }^{*} \mathrm{Mod}\right)$ by the local duality. In particular, $R \operatorname{Hom}_{k[\Delta]}\left(M^{\bullet}, \omega_{k[\Delta]}^{\bullet}\right)$ belongs to $D_{\mathrm{Sq}(\Delta)}^{b}\left({ }^{*} \mathrm{Mod}\right)$, and we can define $R \operatorname{Hom}_{k[\Delta]}\left(M^{\bullet}, \omega_{k[\Delta]}^{\bullet}\right)^{+} \in D^{b}(\operatorname{Sh}(B))$.

If $M \in \operatorname{Sq}(\Delta)$ and $j:|\Delta| \rightarrow B$ is the embedding map, then $\operatorname{Supp}\left(M^{+}\right) \subset|\Delta|$ and $j_{*} j^{*} M^{+} \cong M^{+}$. Since $j_{*}\left(=j_{!}\right): \operatorname{Sh}(|\Delta|) \rightarrow \operatorname{Sh}(B)$ is an exact functor in this case, it can be extended to the functor $j_{*}: D^{b}(\operatorname{Sh}(|\Delta|)) \rightarrow D^{b}(\operatorname{Sh}(B))$.

Theorem 4.2. With the above notation, for $M^{\bullet} \in D^{b}(\operatorname{Sq}(\Delta))$, we have

$$
R \mathcal{H o m}_{\mathrm{Sh}(|\Delta|)}\left(j^{*}\left(M^{\bullet}\right)^{+}, \mathcal{D}_{|\Delta|}^{\bullet}\right) \cong j^{*}\left(R \operatorname{Hom}_{k[\Delta]}\left(M^{\bullet}, \omega_{k[\Delta]}^{\bullet}\right)^{+}\right)
$$

in $D^{b}(\operatorname{Sh}(|\Delta|))$.

Proof. In $D^{b}(\operatorname{Sh}(B))$, we have the following isomorphisms.

$$
\begin{aligned}
& j_{*} R \mathcal{H o m}_{\mathrm{Sh}(|\Delta|)}\left(j^{*}\left(M^{\bullet}\right)^{+}, \mathcal{D}_{|\Delta|}^{\bullet}\right) \\
& \cong j_{*} R \mathcal{H o m}_{\mathrm{Sh}(|\Delta|)}\left(j^{*}\left(M^{\bullet}\right)^{+}, j^{!} \mathcal{D}_{B}^{\bullet}\right) \\
& \cong R \mathcal{H o m}_{\mathrm{Sh}(B)}\left(j_{*} j^{*}\left(M^{\bullet}\right)^{+}, \mathcal{D}_{B}^{\bullet}\right) \quad \text { (by [I, VII. Theorem 5.2]) } \\
& \cong R \mathcal{H o m}_{\mathrm{Sh}(B)}\left(\left(M^{\bullet}\right)^{+}, \text {or }_{B}[n-1]\right) \\
& \left.\cong R \operatorname{Hom}_{k[Q]}\left(M^{\bullet}, K_{k[Q]}[n-1]\right)^{+} \quad \text { (by Lemma } 4.1(3)\right) \\
& \cong R \operatorname{Hom}_{k[\Delta]}\left(M^{\bullet}, \omega_{k[\Delta]}^{\bullet}\right)^{+} \text {. }
\end{aligned}
$$

Hence $j_{*} R \mathcal{H o m}_{\operatorname{Sh}(|\Delta|)}\left(j^{*}\left(M^{\bullet}\right)^{+}, \mathcal{D}_{|\Delta|}^{\bullet}\right) \cong R \operatorname{Hom}_{k[\Delta]}\left(M^{\bullet}, \omega_{k[\Delta]}^{\bullet}\right)^{+}$. Applying $j^{*}$ to the both sides of this isomorphism, we have the expected isomorphism.

Corollary 4.3. With the above notation, we have $\mathcal{D}_{|\Delta|}^{\bullet} \cong j^{*}\left(\omega_{k[\Delta]}^{\bullet}\right)^{+}$. 
Proof.

$$
\mathcal{D}_{|\Delta|}^{\bullet} \cong R \mathcal{H o m}_{\operatorname{Sh}(|\Delta|)}\left(\underline{k}_{|\Delta|}, \mathcal{D}_{|\Delta|}^{\bullet}\right) \cong j^{*}\left(R \operatorname{Hom}_{k[\Delta]}\left(k[\Delta], \omega_{k[\Delta]}^{\bullet}\right)^{+}\right) \cong j^{*}\left(\omega_{k[\Delta]}^{\bullet}\right)^{+}
$$

Proposition 4.4. Let $\Delta, \Sigma \subset L$ be order ideals with $\Delta \supset \Sigma$, and $j$ the embedding map from $Z:=|\Delta|-|\Sigma|$ to $B$. Then

$$
\mathcal{D}_{Z}^{\bullet} \cong j^{*}\left(R \mathcal{H o m}_{k[Q]}\left(I_{\Delta / \Sigma}, \omega_{k[Q]}^{\bullet}\right)^{+}\right),
$$

where $I_{\Delta / \Sigma}:=I_{\Sigma} / I_{\Delta}$.

Proof. In $D^{b}(\operatorname{Sh}(B))$, we have the following isomorphisms.

$$
\begin{aligned}
& R \operatorname{Hom}_{k[Q]}\left(I_{\Delta / \Sigma}, \omega_{k[Q]}^{\bullet}\right)^{+} \\
& \cong R \mathcal{H o m}_{\mathrm{Sh}(B)}\left(j ! \underline{k}_{Z}, \mathcal{D}_{B}^{\bullet}\right) \quad \text { (by Theorem 4.2) } \\
& \cong R j_{*} R \mathcal{H} \operatorname{om}_{\operatorname{Sh}(Z)}\left(\underline{k}_{Z}, j ! \mathcal{D}_{B}^{\bullet}\right) \quad \text { (by [I, VII. Theorem 5.2]) } \\
& \cong R j_{*} R \mathcal{H} \operatorname{Hom}_{\mathrm{Sh}(Z)}\left(\underline{k}_{Z}, \mathcal{D}_{Z}^{\bullet}\right) \\
& \cong R j_{*} \mathcal{D}_{Z}^{\bullet} \text {. }
\end{aligned}
$$

Hence $R \operatorname{Hom}_{k[Q]}\left(I_{\Delta / \Sigma}, \omega_{k[Q]}^{\bullet}\right)^{+} \cong R j_{*} \mathcal{D}_{Z}^{\bullet}$. Applying $j^{*}$ to the both sides of this isomorphism, we have the expected isomorphism. In fact, since the functor $j^{*} j_{*}: \operatorname{Sh}(Z) \rightarrow \operatorname{Sh}(Z)$ is natural equivalent to the identity functor, we have $j^{*} R j_{*} \cong j^{*} j_{*} \cong \mathrm{Id}$ as an endofunctor on $D^{b}(\operatorname{Sh}(Z))$.

In our context, the notion of a Buchsbaum ring is natural and important. The original definition of a Buchsbaum ring (see [SV]) is slightly complicated, but for $k[\Delta]$, we have a simple criterion.

Lemma 4.5. Let $A=\bigoplus_{i>0} A_{i}$ be a noetherian $\mathbb{N}$-graded commutative ring with the graded maximal ideal $\mathfrak{m}=\bigoplus_{i>0} A_{i}$ (thus $A_{0}=k$ is a field). Let $M$ be a finitely generated graded $A$-module of dimension $r$. If there is some $s \in \mathbb{Z}$ such that $\left[H_{\mathfrak{m}}^{i}(M)\right]_{t}=0$ for all $i<r$ and $t \neq s$, then $M$ is a Buchsbaum A-module.

If $A$ is generated by $A_{1}$ as a $k$-algebra, the above fact is a special case of the well-known result [SV, I. Proposition 3.10]. Even in the general case, this fact was essentially pointed out in [SS].

Proof. Note that $A$ has a graded normalized dualizing complex $I_{A}^{\bullet}$. Set $N^{\bullet}:=$ $\tau_{-r} \operatorname{Hom}_{A}^{\bullet}\left(M, I_{A}^{\bullet}\right)$. Here, for a complex $C^{\bullet}, \tau_{-r} C^{\bullet}$ is the truncated complex

$$
\cdots \longrightarrow 0 \longrightarrow \operatorname{lm}\left(C^{-r} \rightarrow C^{-r+1}\right) \longrightarrow C^{-r+1} \longrightarrow C^{-r+2} \longrightarrow \cdots
$$

We have $H^{i}\left(N^{\bullet}\right)=0$ for all $i \leq-r$, and $H^{i}\left(N^{\bullet}\right)$ is the graded $k$-dual of $H_{\mathfrak{m}}^{-i}(M)$ for all $i>-r$ by the local duality. So the cohomologies of $N^{\bullet}$ are concentrated in the degree $-s$ components. By [SV, II.Theorem 4.1], it suffices to prove that $N^{\bullet}$ is isomorphic to a complex of $k$-vector spaces in the derived category of graded $A$ modules. For a graded $A$-module $N$ and an integer $t$, set $N_{\geq t}:=\bigoplus_{i \geq t} N_{i}$. Then chain maps $N_{\geq-s}^{\bullet} \rightarrow N^{\bullet}$ and $N_{\geq-s}^{\bullet} \rightarrow N_{\geq-s}^{\bullet} / N_{\geq-s+1}^{\bullet}$ are quasi-isomorphisms. 
Thus, in the derived category, $N^{\bullet}$ is isomorphic to $N_{\geq-s}^{\bullet} / N_{\geq-s+1}^{\bullet}$, which is a complex of $k$-vector spaces.

Corollary 4.6 (c.f. [Y2, Corollary 3.8]). Let $M$ be a squarefree $k[Q]$-module of dimension $r$. Then the following are equivalent.

(a) $M$ is a Buchsbaum module.

(b) $\operatorname{dim}_{k} H_{\mathfrak{m}}^{i}(M)<\infty$ for all $i \neq r$.

(c) $\left[H_{\mathfrak{m}}^{i}(M)\right]_{\mathbf{a}}=0$, if $i \neq r$ and $\mathbf{a} \neq 0$.

Proof. The implication (a) $\Rightarrow(\mathrm{b})$ is a basic property of Buchsbaum modules. The $\mathbb{Z}^{n}$-graded $k$-dual of $H_{\mathfrak{m}}^{i}(M)$ is the squarefree module $\operatorname{Ext}_{k[Q]}^{n-i}\left(M, K_{k[Q]}\right)$. Hence $\operatorname{dim}_{k} H_{\mathfrak{m}}^{i}(M)<\infty$ if and only if $H_{\mathfrak{m}}^{i}(M)=\left[H_{\mathfrak{m}}^{i}(M)\right]_{0}$. So we have (b) $\Leftrightarrow$ (c). The implication (c) $\Rightarrow$ (a) follows from Lemma 4.5 .

Corollary 4.7. Let $\Delta \subset L$ be an order ideal with $d=\operatorname{dim}|\Delta|(\operatorname{so} \operatorname{dim} k[\Delta]=$ $d+1)$. The following are equivalent.

(a) $k[\Delta]$ is a Buchsbaum ring.

(b) $\mathcal{H}^{i}\left(\mathcal{D}_{|\Delta|}^{\bullet}\right)=0$ for all $i \neq-d$.

(c) $H_{i}(|\Delta|,|\Delta|-\{p\} ; k)=0$ for all $i<d$ and all $p \in|\Delta|$.

In particular, the Buchsbaum property of $k[\Delta]$ is a topological property of $|\Delta|$ (i.e., depends only on the topology of $|\Delta|$ and $\operatorname{char}(k))$.

Proof. We have $\mathcal{H}^{i}\left(\mathcal{D}_{|\Delta|}^{\bullet}\right) \cong j^{*}\left(H^{i}\left(\omega_{k[\Delta]}^{\bullet}\right)^{+}\right) \cong j^{*}\left(\operatorname{Ext}_{k[Q]}^{i}\left(k[\Delta], \omega_{k[Q]}^{\bullet}\right)^{+}\right)$by Corollary 4.3, where $j:|\Delta| \rightarrow B$ is the embedding map. Thus $\mathcal{H}^{i}\left(\mathcal{D}_{|\Delta|}^{\bullet}\right)=0$ if and only if $\operatorname{dim}_{k} \operatorname{Ext}_{k[Q]}^{i}\left(k[\Delta], \omega_{k[Q]}^{\bullet}\right)=\operatorname{dim}_{k} H_{\mathfrak{m}}^{-i+1}(k[\Delta])<\infty$. So (b) is equivalent to (a). The equivalence (b) $\Leftrightarrow$ (c) must be obvious for algebraic topologists. But the equivalence (c) $\Leftrightarrow$ (a) ( $\Leftrightarrow$ (b)) also follows from Theorem 3.5. In fact, if $0 \neq \mathbf{a} \in \mathbb{N}^{n}$, we have $\left[H_{\mathfrak{m}}^{i+1}(k[\Delta])\right]_{-\mathbf{a}} \cong H_{i}(|\Delta|,|\Delta|-\{p\} ; k)$ for $p \in|\operatorname{supp}(\mathbf{a})|^{\circ}$, as we have seen in Remark 3.6.

Remark 4.8. The implication (b) $\Rightarrow$ (a) of Corollary 4.7 does not hold for a locally closed subset $Z:=|\Delta|-|\Sigma|$ and its squarefree module $I_{\Delta / \Sigma}:=I_{\Sigma} / I_{\Delta}$, while we have Proposition 4.4. Since

$$
R \operatorname{Hom}_{k[Q]}\left(I_{\Delta / \Sigma}, \omega_{k[Q]}^{\bullet}\right)^{+} \cong R j_{*} \mathcal{D}_{Z}^{\bullet}
$$

by the proof of Proposition 4.4, $I_{\Delta / \Sigma}$ is a Buchsbaum module of dimension $d+1$ if and only if $R^{i} j_{*} \mathcal{D}_{Z}^{\bullet}=0$ for all $i \neq-d$. These conditions are stronger than the condition that $\mathcal{H}^{i}\left(\mathcal{D}_{Z}^{\bullet}\right)=0$ for all $i \neq-d$.

For example, consider a polynomial ring $k[x, y, z]$, and simplicial complexes $\Delta=2^{\{x, y, z\}}$ and $\Sigma=\{\{x\}, \emptyset\}$. Then $Z$ is a manifold with boundary (in fact, $\left.Z \cong \mathbb{R}_{+}^{2}\right)$, and $\mathcal{H}^{i}\left(\mathcal{D}_{Z}^{\bullet}\right)=0$ for all $i \neq-2$. But $I_{\Delta / \Sigma}=(y, z)$ is not a Buchsbaum module. In this case, $R^{i} j_{*} \mathcal{D}_{Z}^{\bullet} \neq 0$ for $i=-1,-2$.

Since $\operatorname{Supp}\left(R^{i} j_{*} \mathcal{D}_{Z}^{\bullet}\right) \subset \bar{Z}=|\Delta|$, it suffices to check $R^{i} h_{*} \mathcal{D}_{Z}^{\bullet}$ to see the vanishing of $R^{i} j_{*} \mathcal{D}_{Z}^{\bullet}$, where $h: Z \rightarrow|\Delta|$ is the embedding map. That is, $I_{\Delta / \Sigma}$ is a Buchsbaum module of dimension $d+1$ if and only if $R^{i} h_{*} \mathcal{D}_{Z}^{\bullet}=0$ for all 
$i \neq-d$. Hence the Buchsbaum property of $I_{\Delta / \Sigma}$ is a topological property of the pair $(|\Delta|,|\Sigma|)$.

If $|\Delta|$ is a manifold (with or without boundary) of dimension $d$, then we have $\mathcal{D}_{|\Delta|}^{\bullet} \cong \operatorname{or}_{|\Delta|}[d]$ and $k[\Delta]$ is a Buchsbaum ring of dimension $d+1$. By Corollary 4.3 , we have $j^{*}\left(K_{k[\Delta]}\right)^{+} \cong o r_{|\Delta|}$, where $K_{k[\Delta]}:=\operatorname{Ext}_{k[Q]}^{n-d-1}\left(k[\Delta], K_{k[Q]}\right)$ is the canonical module of $k[\Delta]$.

Let $(A, \mathfrak{m})$ be a Buchsbaum local ring of dimension $d+1$ admitting a canonical modules $K_{A}$. Then [SV, II. Theorem 4.9] states that

$$
H_{\mathfrak{m}}^{i}\left(K_{A}\right) \cong \operatorname{Hom}_{A}\left(H_{\mathfrak{m}}^{d-i+2}(A), E(A / \mathfrak{m})\right) \text { for all } 2 \leq i \leq d,
$$

where $E(A / \mathfrak{m})$ is the injective hull of $A / \mathfrak{m}$. We will see that this duality corresponds to the Poincaré duality in our context.

Assume that $k[\Delta]$ is a Buchsbaum ring of dimension $d+1$ (thus $\operatorname{dim}|\Delta|=d)$. Then we have

$$
\left[H_{\mathfrak{m}}^{i}\left(\left(K_{k[\Delta]}\right)_{>0}\right)\right]_{0} \cong\left[H_{\mathfrak{m}}^{d-i+2}\left(k[\Delta]_{>0}\right)^{\vee}\right]_{0} \quad \text { for all } 1 \leq i \leq d+1 .
$$

(When $2 \leq i \leq d$, this is just a $\mathbb{Z}^{n}$-graded version of [SV, II. Theorem 4.9]. We leave the case when $i=1, d+1$ for the reader as an easy exercise.) By Theorem 3.3,

$$
\left[H_{\mathfrak{m}}^{i}\left(\left(K_{k[\Delta]}\right)_{>0}\right)\right]_{0} \cong H^{i-1}\left(|\Delta|,\left(K_{k[\Delta]}\right)^{+}\right) \cong H^{i-1}\left(|\Delta|, o r_{|\Delta|}\right)
$$

and

$$
\left[H_{\mathfrak{m}}^{d-i+2}\left(k[\Delta]_{>0}\right)\right]_{0} \cong H^{d-i+1}\left(|\Delta|, \underline{k}_{|\Delta|}\right) \cong H^{d-i+1}(|\Delta| ; k)
$$

for all $1 \leq i \leq d+1$. So (4.2) also follows from the Poincaré duality

$$
H^{i}\left(|\Delta|, \text { or }_{|\Delta|}\right) \cong H^{j}(|\Delta| ; k)^{\vee} \text { for all } i, j \text { with } i+j=d .
$$

Note that $|\Delta|$ is an orientable manifold (i.e., a manifold with $\underline{k}_{|\Delta|} \cong$ or $r_{|\Delta|}$ ) if and only if $k[\Delta]$ is a Buchsbaum ring with $\left(K_{k[\Delta]}\right)_{>0} \cong k[\Delta]_{>0}$. In this case, (4.2) corresponds to the most familiar form of the Poincaré duality. We also remark that if $|\Delta|$ is an orientable manifold of dimension $d$ then $\operatorname{dim}_{k}\left[H_{\mathfrak{m}}^{d+1}(k[\Delta])\right]_{0}$ equals the number of the connected components of $|\Delta|$. When $|\Delta|$ is a connected manifold, $|\Delta|$ is orientable if and only if $\operatorname{dim}_{k}\left[H_{\mathfrak{m}}^{d+1}(k[\Delta])\right]_{0}=1$. In this case, $K_{k[\Delta]} \cong k[\Delta]$.

Let $\mathrm{Sq}_{+}(\Delta)$ be the full subcategory of Sq consisting of squarefree $k[\Delta]$ modules $M$ with $M_{0}=0$. For a while, let $M^{\bullet}$ be an object of $D^{b}\left(\operatorname{Sq}_{+}(\Delta)\right)$.

For $M^{\bullet} \in D^{b}\left(\mathrm{Sq}_{+}(\Delta)\right)$, by the local duality and Theorem 3.3, we have

$$
\left[\operatorname{Ext}_{k[\Delta]}^{-i}\left(M^{\bullet}, \omega_{k[\Delta]}^{\bullet}\right)^{\vee}\right]_{0} \cong\left[R^{i+1} \Gamma_{\mathfrak{m}}\left(M^{\bullet}\right)\right]_{0} \cong R^{i} \Gamma\left(B,\left(M^{\bullet}\right)^{+}\right) \cong R^{i} \Gamma\left(|\Delta|, j^{*}\left(M^{\bullet}\right)^{+}\right) \text {. }
$$

On the other hand, we have $\operatorname{Ext}_{\operatorname{Sh}(|\Delta|)}^{-i}\left(j^{*}\left(M^{\bullet}\right)^{+}, \mathcal{D}_{|\Delta|}^{\bullet}\right)^{\vee} \cong R^{i} \Gamma\left(|\Delta|, j^{*}\left(M^{\bullet}\right)^{+}\right)$ by the Poincaré-Verdier duality ([I, V, 2.1]). Thus

$$
\operatorname{Ext}_{\operatorname{Sh}(|\Delta|)}^{i}\left(j^{*}\left(M^{\bullet}\right)^{+}, \mathcal{D}_{|\Delta|}^{\bullet}\right) \cong\left[\operatorname{Ext}_{k[\Delta]}^{i}\left(M^{\bullet}, \omega_{k[\Delta]}^{\bullet}\right)\right]_{0} .
$$


We can give another proof of (4.3). Let $P^{\bullet} \rightarrow M^{\bullet}$ be a projective resolution in Sq. Since $M^{\bullet} \in D^{b}\left(\mathrm{Sq}_{+}(\Delta)\right)$, we may assume that each component of $P^{\bullet}$ is a direct sum of copies of $J_{F}$ for various $\{0\} \neq F \in L$. If $F \neq\{0\}$, then $\operatorname{Supp}\left(\left(J_{F}\right)^{+}\right)=U_{F} \cong \mathbb{R}^{n-1}$ or $\mathbb{R}_{+}^{n-1}$ and $\operatorname{Ext}_{\operatorname{Sh}(B)}^{i}\left(\left(J_{F}\right)^{+}\right.$, or $\left._{B}\right)=$ $H^{n-1-i}\left(B,\left(J_{F}\right)^{+}\right)=H_{c}^{n-1-i}\left(U_{F} ; k\right)=0$ for all $i \neq 0$. So we can compute $\operatorname{Ext}_{\operatorname{Sh}(B)}^{i}\left(\left(M^{\bullet}\right)^{+}\right.$, or $\left._{B}\right)$ using $\left(P^{\bullet}\right)^{+}$, and we have the following.

$$
\begin{aligned}
& \operatorname{Ext}_{\operatorname{Sh}(|\Delta|)}^{i}\left(j^{*}\left(M^{\bullet}\right)^{+}, \mathcal{D}_{|\Delta|}^{\bullet}\right) \\
\cong & \operatorname{Ext}_{\operatorname{Sh}(B)}^{i}\left(\left(M^{\bullet}\right)^{+}, \mathcal{D}_{B}^{\bullet}\right) \quad(\text { by }[\text { I, VII. Theorem 3.1]) } \\
\cong & \operatorname{Ext}_{\operatorname{Sh}(B)}^{i}\left(\left(M^{\bullet}\right)^{+}, \text {or }_{B}[n-1]\right) \\
\cong & H^{i}\left(\operatorname{Hom}_{\operatorname{Sh}(B)}\left(\left(P^{\bullet}\right)^{+},\left(K_{k[Q]}\right)^{+}[n-1]\right)\right) \\
\cong & H^{i}\left(\left[\operatorname{Hom}_{k[Q]}\left(P^{\bullet}, K_{k[Q]}[n-1]\right)\right]_{0}\right) \quad\left(\text { since } P^{\bullet} \in D^{b}\left(\operatorname{Sq}_{+}\right)\right) \\
\cong & {\left[\operatorname{Ext}_{k[Q]}^{i}\left(M^{\bullet}, K_{k[Q]}[n-1]\right)\right]_{0} } \\
\cong & {\left[\operatorname{Ext}_{k[\Delta]}^{i}\left(M^{\bullet}, \omega_{k[\Delta]}^{\bullet}\right)\right]_{0} . }
\end{aligned}
$$

Finally, we study the Cohen-Macaulay property of $k[\Delta]$ and $I_{\Delta / \Sigma}$. If $\operatorname{dim} k[\Delta] \leq$ 1 , then $k[\Delta]$ is always Cohen-Macaulay. So we may assume that $\operatorname{dim} k[\Delta] \geq 2$. The same thing is true for $I_{\Delta / \Sigma}$. When $k[Q]$ is a polynomial ring, the next result is a well-known theorem of Munkres.

Theorem 4.9 (c.f. [M, Y3]). Let $\Delta \subset L$ be an order ideal with $d:=\operatorname{dim}|\Delta| \geq 1$ (i.e., $\operatorname{dim} k[\Delta]=d+1 \geq 2$ ). Then the following are equivalent.

(a) $k[\Delta]$ is a Cohen-Macaulay ring of dimension $d+1$,

(b) $\tilde{H}_{i}(|\Delta| ; k)=H_{i}(|\Delta|,|\Delta|-\{p\} ; k)=0$ for all $i<d$ and all $p \in|\Delta|$,

(c) $\mathcal{H}^{i}\left(\mathcal{D}_{|\Delta|}^{\bullet}\right)=0$ for all $i \neq-d, H^{i} \Gamma\left(|\Delta|, \mathcal{D}_{|\Delta|}^{\bullet}\right)=0$ for all $i \neq-d$, 0 , and $H^{0} \Gamma\left(|\Delta|, \mathcal{D}_{|\Delta|}^{\bullet}\right) \cong k$.

In particular, the Cohen-Macaulay property of $k[\Delta]$ is a topological property of $|\Delta|$.

Proof. The equivalence between (a) and (b) has been proved in [M, Y3]. Recall that $H_{i}(|\Delta|,|\Delta|-\{p\} ; k)=0$ for all $i<d$ and all $p \in|\Delta|$ if and only if $\mathcal{H}^{i}\left(\mathcal{D}_{|\Delta|}^{\bullet}\right)=0$ for all $i \neq-d$. Since $H^{-i} \Gamma\left(|\Delta|, \mathcal{D}_{|\Delta|}^{\bullet}\right) \cong H^{i}(|\Delta| ; k)^{\vee}$, (b) and (c) are equivalent.

Proposition 4.10. Let $\Delta, \Sigma \subset L$ be order ideals with $\Delta \supset \Sigma \neq \emptyset$, and $h$ the embedding map from $Z:=|\Delta|-|\Sigma|$ to $|\Delta|$.

(a) $I_{\Delta / \Sigma}$ is a Cohen-Macaulay module of dimension $d+1$,

(b) $R^{i} h_{*} \mathcal{D}_{Z}^{\bullet}=H^{i} \Gamma\left(Z, \mathcal{D}_{Z}^{\bullet}\right)=0$ for all $i \neq-d$.

In particular, the Cohen-Macaulay property of $I_{\Delta / \Sigma}$ is a topological property of the pair $(|\Delta|,|\Sigma|)$.

Proof. $I_{\Delta / \Sigma}$ is Cohen-Macaulay if and only if it is Buchsbaum and $\left[H_{\mathfrak{m}}^{i}\left(I_{\Delta / \Sigma}\right)\right]_{0}=$ 0 for all $i \neq d+1$. As we have seen in Remark 4.8, $I_{\Delta / \Sigma}$ is Buchsbaum if and 
only if $R^{i} h_{*} \mathcal{D}_{Z}^{\bullet}=0$ for all $i \neq-d$. Since $I_{\Delta / \Sigma} \in \mathrm{Sq}_{+}$, we have $\left[H_{\mathfrak{m}}^{i+1}\left(I_{\Delta / \Sigma}\right)\right]_{0} \cong$ $H^{i}\left(B,\left(I_{\Delta / \Sigma}\right)^{+}\right) \cong H_{c}^{i}(Z ; k) \cong H^{-i} \Gamma\left(Z, \mathcal{D}_{Z}^{\bullet}\right)^{\vee}$ for all $i$. So we are done.

\section{Acknowledgments}

I am grateful to Professor Ezra Miller. This paper grew out of the discussion with him about his paper [M]. I also thank Professor Takesi Kawasaki for giving me useful comments on (graded) Buchsbaum modules.

\section{References}

[BH] W. Bruns and J. Herzog, Cohen-Macaulay rings, revised edition, Cambridge University Press, 1998.

[GW] S. Goto and K. Watanabe, On graded rings. II. ( $\mathbf{Z}^{n}$-graded rings). Tokyo J. Math. 1 (1978), 237-261.

[H] R. Hartshorne, Residues and duality, Lectures Notes in Math. 20, Springer-Verlag, 1966.

[I] B. Iversen, Cohomology of sheaves. Springer-Verlag, 1986.

[M] E. Miller, Cohen-Macaulay quotients of normal affine semigroup rings via irreducible resolutions, Math. Research Letters 9 (2002), 117-128.

[RWY] V. Reiner, V. Welker and K. Yanagawa. Local cohomology of Stanley-Reisner rings with supports in general monomial ideals, J. Algebra 244 (2001), 706-736.

[SS] U. Schäfer and P. Schenzel, Dualizing complexes of affine semigroup rings, Trans. Amer. Math. Soc. 322 (1990), 561-582.

[Sta] R. Stanley, Combinatorics and commutative algebra, 2nd ed, Progress in Mathematics, 41, (1996).

[SV] S. Stuckrad and W. Vogel, Buchsbaum rings and applications, Springer-Verlag, 1986.

[Y1] K. Yanagawa, Alexander duality for Stanley-Reisner rings and squarefree $\mathbb{N}^{n}$-graded modules, J. Algebra 225 (2000), 630-645.

[Y2] _ Sheaves on finite posets and modules over normal semigroup rings, J. Pure and Appl. Algebra 161 (2001), 341-366.

[Y3] _ Squarefree modules and local cohomology modules at monomial ideals, in "Local Cohomology and Its Applications (G. Lyubeznik, ed.)," pp. 207-231, Dekker, 2002.

Department of Mathematics, Graduate School of Science, Osaka University, ToyONAKA, OSAKA 560-0043, JAPAN

E-mail address: yanagawa@math.sci.osaka-u.ac.jp 